\title{
Universiteit
}

Leiden

The Netherlands

\section{Exploring video games that invoke curiosity}

Gómez Maureira, M.A.; Kniestedt, I.

\section{Citation}

Gómez Maureira, M. A., \& Kniestedt, I. (2019). Exploring video games that invoke curiosity. Entertainment Computing, 32. doi:10.1016/j.entcom.2019.100320

Version: $\quad$ Publisher's Version

License: $\quad$ Licensed under Article 25fa Copyright Act/Law (Amendment Taverne)

Downloaded from: https://hdl.handle.net/1887/3217498

Note: To cite this publication please use the final published version (if applicable). 


\title{
Exploring video games that invoke curiosity
}

\author{
Marcello A. Gómez-Maureira ${ }^{\mathrm{a}}$, Isabelle Kniestedt ${ }^{\mathrm{b}}$ \\ ${ }^{\mathrm{a}}$ Leiden Institute of Advanced Computer Science, Leiden, the Netherlands \\ ${ }^{\mathrm{b}}$ Delft University of Technology, Delft, the Netherlands
}

A R T I C L E I N F O

\section{Keywords:}

Game user research

Curiosity research

Game analysis

\begin{abstract}
A B S T R A C T
Curiosity is considered an important aspect of human life, but understanding the circumstances that cause a person to become curious poses a challenge for research. This paper proposes video games as a stimulus for the experimental study of curiosity. For this purpose, we conducted a survey with the goal of assessing what video game titles and genres could be considered reliable instruments for invoking curiosity. To involve different types of curiosity, we included the Five-Dimensional Curiosity Scale (5DC) questionnaire. The survey was completed by 113 participants, and resulted in 301 game suggestions that warrant further analysis. Exploration and Social Simulation games in particular were found to rank high in triggering curiosity. To explain this result, we present a first analysis of potential game patterns that help trigger curiosity within these genres.
\end{abstract}

\section{Introduction}

Curiosity plays a crucial role in many aspects of human life. It is a sign of intrinsic motivation to learn and explore [1-3]. In education and research, curiosity is frequently credited as one of the most important factors for human progress [4-6]. Since curiosity is presumed to involve both behavioural and emotional components [5], studying it is challenging. On the other hand, progress has been made in recent work in establishing definitions of curiosity and psychometric instruments to measure it. As a result, there is growing interest in the applied use of what has been learned about curiosity, such as to improve teaching methods $[7,8]$ and the design of video games $[9,10]$. Video games, here understood as digitally-mediated systems for structured play, provide multi-faceted environments that can stimulate curiosity [11,12]. The question of which games accomplish this and how is what we seek to answer in this paper. There is a lack of specific knowledge on which elements of games stand out in their ability to invoke curiosity. Knowing this would allow for more in-depth analysis of the methods that existing games use to make players curious.

In this study we thus aim to lay the groundwork for filling this gap. We present the results from an exploratory survey involving 113 participants. In the survey, we asked players to rank well-known games according to how curious they felt while playing them. We further inquired which game titles made them curious in the past, using established dimensions of curiosity as prompts [13]. A total of 301 games were mentioned by participants and were then categorized according to a list of predefined game genres. This categorization allowed us to analyze patterns within the varied collection. With this, we examine what games and game genres are successful in invoking curiosity. Participants also filled in the 5DC questionnaire [13] which measures curiosity on five constituent dimensions. With this data, we examined whether there is a connection between an individual's tendency to become curious and the game genres that invoke curiosity.

The primary contribution of this study takes the form of an informed selection of games and game genres that warrant closer analysis in regards to how the elicitation of curiosity may be designed for within a game. Additionally, two genres that ended up highest in the ranking, Exploration and Social Simulation, are discussed in more detail. Based on the collection of games suggested by participants, we formulate hypotheses to explain how these genres are successful in invoking curiosity. Due to its exploratory nature, this work does not aim at formalizing a generalizable theory. However, we believe that it is an important step in exploring curiosity within different games and a basis for further work in this direction.

\section{Related work}

Most research efforts regarding curiosity have taken place in the fields of philosophy $[14,4]$ and psychology $[15,16]$. Inherent in this past is the fact that definitions of curiosity vary, ranging from accounts of human aspirations to describing it as instigating stimulant for interaction with the environment.

In this study we understand curiosity as an intrinsic motivation for pursuing new knowledge and experiences that is accompanied by pleasure and excitement. This understanding of curiosity is based on a meta review of academic articles which aimed to find commonalities in

E-mail addresses: m.a.gomez.maureira@liacs.leidenuniv.nl (M.A. Gómez-Maureira), i.kniestedt@tudelft.nl (I. Kniestedt). 
prior research [5]. In the review, the author discusses different research lenses through which curiosity has been studied. These lenses do not necessarily contradict each other, but focus on different aspects of curiosity. One view of curiosity, for example, is to consider it a primal drive that requires satisfaction $[16,17]$, not unlike satisfying hunger [4]. Another view is to see curiosity as a need to fill gaps in knowledge [6], requiring both existing knowledge to be aware of such a gap, as well as the evaluation that the gap is neither too large nor too insignificant to be filled [18]. Important for our study is the differentiation between curiosity as a state and curiosity as a trait. The former is the 'in-the-moment' drive for exploratory behaviour and its emotional impact [6]. Trait curiosity, on the other hand, is an individual's tendency or disposition to become curious and is considered a relatively stable personality trait [19]. It should be noted that studies have shown an influential relationship between trait and state curiosity [20-22].

Most of the existing work in quantifying curiosity is concerned with measuring trait curiosity $[23,1]$ or related personality traits, such as intrinsic motivation $[24,25]$ or sensation seeking [26]. To quantify curiosity in our study, we follow the curiosity model proposed by Kashdan et al. which suggests the involvement of five dimensions to describe an individual's disposition to become curious [13]. The individual dimensions were selected based on preceding work and validated through three surveys. The result of their study is the 'Five-Dimensional Curiosity Scale' (5DC) which quantifies trait curiosity through a validated questionnaire.

Games are a promising area for researching curiosity, as they introduce further related concepts that can help to gain a better understanding. Costikyan's work regarding the role of uncertainty in games, for example, involves curiosity and describes it as an important motivator to engage in gameplay [27]. For Klimmt [28], curiosity is part of a conceptual model for player engagement, i.e. the reason why people choose to play games. Studies into player profiling seek to establish player archetypes that involve personality traits and motivations, including curiosity [10]. Interestingly, such player archetypes can directly mirror aspects of Kashdan et al.'s aforementioned curiosity model. The BrainHex model [29] features seven archetypes that match the characteristics of different dimensions of curiosity. The 'daredevil' archetype, for example, is defined by taking pleasure in taking and overcoming risks, matching the 'thrill seeking' dimension in the 5DC. In these cases, however, curiosity is not studied on its own but mentioned as a contributing factor.

Games have also been proposed as instruments for measuring curiosity, as was done in a study from 2012 to measure scientific curiosity in children [12]. In this experiment, the performance of players within an exploration game was used as a behavioural measure instead of relying on self-report through a questionnaire.

An improved understanding of curiosity also benefits efforts in understanding player experience and can inform game development. Research by To et al. [9] investigated how game designers can elicit the curiosity of players. In their study, they follow a model of curiosity [30] that distinguishes between different triggers of curiosity. This approach is particularly useful for creating generalizable design guidelines, as it gives game designers a range of possible design interventions for invoking curiosity. Overall, existing research shows that games are able to elicit curiosity, and that this ability is useful for both research and development. We are not aware of work that investigates which games stand out as being particularly capable of invoking curiosity, and thus aim to provide such insights with this study.

\section{Exploratory survey}

As an initial step in the study of game elements that invoke curiosity, we set out to establish the types of games that make players feel curious. By asking players to rank well-known games in terms of how curious they felt while playing them, as well as suggest games that made them feel curious, we aimed to establish a first corpus of games for further study. This section provides a summary of the survey and its key results.

\subsection{Method}

An online survey aimed at people playing video games was distributed through various channels, e.g. Facebook groups connected to gaming and game research. It included the following modules: Demographics, shared selection of games, suggestions by curiosity dimensions, and the 5DC questionnaire. Each of the modules is described below, as well as the formulation of genres to categorize games suggested by participants.

\subsection{1. $5 D C$ questionnaire}

We used the 5DC questionnaire [13] to explore if the individual dimensions of trait curiosity predict the game genres that invoke curiosity in a player. This questionnaire scores curiosity in five dimensions: Joyous Exploration (JE) - being motivated by novelty, Deprivation Sensitivity (DS) - need of resolving, Stress Tolerance (ST) - ability to cope with uncertainty, Social Curiosity (SC) - wanting to know about others, and Thrill Seeking (TS) - enjoyment of anxiety. The questionnaire has been developed by selecting items of existing measures that evaluate interest and curiosity, openness to experience, need for cognition, boredom proneness, and sensation seeking. Individual items were evaluated through three studies with a combined sample size of 3911 participants. Finally, the questionnaire was examined in regards to testretest reliability through a 4-month follow-up, with results being within the range of stable personality traits.

\subsubsection{Shared selection of games}

Players were presented with a list of 15 acclaimed game titles and asked to rank those they had played, in order to explore which games invoked curiosity during play (see Fig. 1). It should be noted that this study is not about how curious people are to play a specific game (e.g. not played yet, but curious to do so), but how curious they felt as part of the gameplay. Questions regarding curiosity were phrased to reflect this focus. By presenting a predefined list, we could collect data on specific game titles that can be considered of solid quality in terms of design. The measure for quality was provided by a game's Metacritic score [31], which itself is comprised of the evaluation of several game critics.

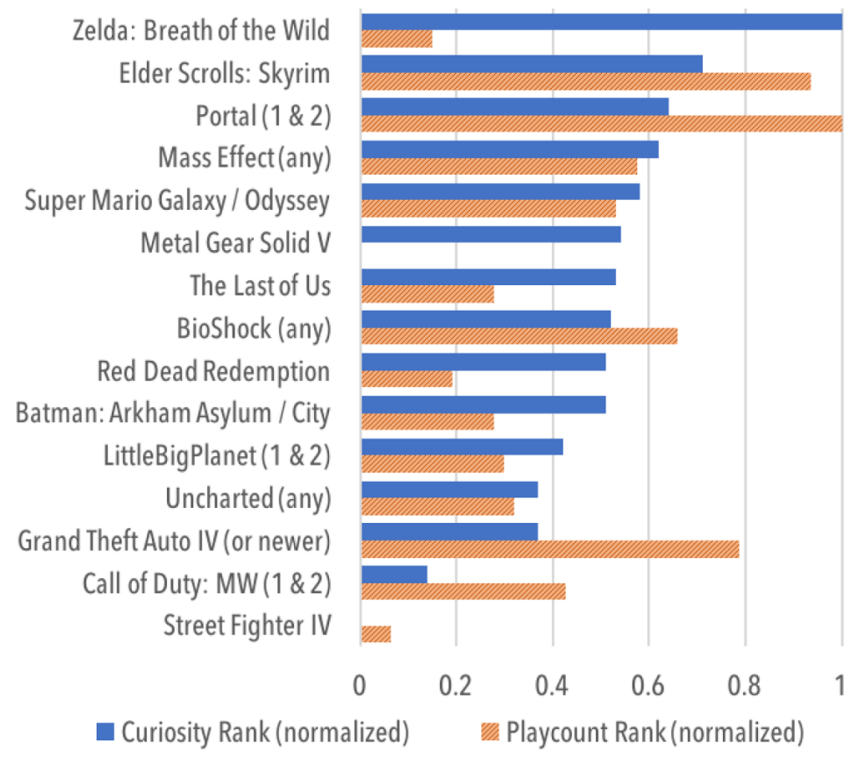

Fig. 1. Shared selection of games ranked by how curious participants felt and how many participants had played them. Values are normalized to $0-1$ for comparison $(0=$ lowest rank, $1=$ highest rank $)$. 
While this measure is based on subjective evaluations, it is a reasonable approximation for choosing games of comparable quality. We took the top 15 games listed on Metacritic, after restricting our selection to games that were released in the last 10 years and combining games of the same series that met that criteria. It could be possible for aspects of games that have developed over the years, e.g. graphical fidelity, to influence curiosity in players. By limiting our selection by time of release, we aimed to make the diverse group of games homogeneous on a technical level and therefore limit bias. The resulting selection involved games with a Metacritic score of 94 or higher (out of 100).

\subsubsection{Suggestions by curiosity dimensions}

Given that we were interested in exploring what game titles should be analysed in regards to their ability to invoke curiosity, we asked participants to suggest game titles as well. In order to consider different dimensions of curiosity, we used five categories for which suggestions could be added (up to two games per category). These were described as "Games that ...": "let me explore or find out new things" (GEXP), "let me solve something" (GSOL), "let me feel safe and stress-free" (GSAF), "let me understand people or let me connect to people" (GCON), and "make me feel excited and alive" (GALI).

We based the category descriptions on the questions that make up the dimensions of the 5DC questionnaire. As a result, the categories should match the five curiosity dimensions: GEXP matching Joyous Exploration, GSOL matching Deprivation Sensitivity, GSAF matching Stress Tolerance, GCON matching Social Curiosity, and GALI matching Thrill Seeking. In addition to suggesting game titles, participants also ranked their own suggested titles in order of how curious they felt while playing them.

\subsubsection{Ranking}

We asked participants to rank the games they had played, rather than rate them on a Likert scale. Reporting about affective constructs is challenging and applying a rating consistently can be particularly difficult [32]. Ranking, on the other hand, allows participants to use the individual items as points of reference. The challenge is then how to evaluate such rankings across participants. Not all people play the same game titles. Likewise, participants may rank varying numbers of games, either because they do not play as many games or because they do not consider them to be invoking curiosity. We thus implemented the TrueSkill rating system [33], developed by Microsoft for ranking and match-making on their Xbox LIVE online platform [34], to analyze the data. TrueSkill uses a Bayesian inference algorithm that updates the score of individual match items (usually representing the skill of players) every time a match is played. Since score-points can be lost, participating in a high number of matches (i.e. a game having been played by many participants) does not necessarily result in a higher ranking. As such, we can use this algorithm to compare items (the individual games) that vary in regards to how often they were mentioned. To use TrueSkill, we paired up all combinations within a ranking to create 'match-ups', taking the rank as deciding factor on which item 'wins' the match. After matching up all possible combinations, we used the resulting score as a measure for both the rank of an item, as well as the relative distance to other items. While the score is an arbitrary number, it can be used in relation to other scores. Items that have relatively similar scores can then be considered closer to equal, while those that differ by wide margins are likely to have 'won' a large number of comparisons. While using the TrueSkill algorithm provides a useful model for ranking items, we cannot evaluate how significant the resulting rank is. To our knowledge there is no statistical test that can estimate how representative the overall rankings are for a larger population. While a different survey design would have remedied this, it would mean to either only include participants that have played the same games, or resort to rating games on a scale.

\subsubsection{Formulation of game genres}

When asking participants to suggest game titles, we can expect a wide range of games. This makes it difficult to explore general patterns, as the number of participants that will have played the same games will be limited. In order to capture the most defining aspects of a game instead, we assigned two game genres to each of the suggested games. The challenge of involving genres is the lack of a shared definition. Genre classifications can originate from multiple motivations, such as easing retrieval of titles, academic efforts of building a taxonomy, or marketing considerations [35]. For this reason we devised a list of 11 game genres based on commercially used genres, but qualified by a statement that defines the genre in our study. Some of the more commonly used genres have been omitted or modified to suit the goals of this study. As an example, "Action" can be a problematic genre, as a large number of games involve fast-paced sequences but may be based on vastly different game mechanics. An additional challenge is that games frequently involve a wide range of game genres. Grand Theft Auto $V$ [36] (GTA $V$ ) lets players shoot virtual characters and race with cars, but also ride a roller-coaster, attend therapy sessions, and solve a murder mystery. By attributing the genres Reflex and Exploration, some nuance is undoubtedly lost. While imperfect, this approach still provides a tentative measure for evaluating which actions performed in a game can be conductive to invoking curiosity. We assigned the following genres:

- Reflex - requires fast reflexes to perform well.

- Exploration - provides spatial or conceptual discovery that is not automatically brought to the attention of the player.

- Puzzle - presents tasks that must be solved through predefined processes.

- Strategy - requires players to plan their actions in advance, taking into consideration available resources.

- $R P G$ - defined by assuming the role of one or more characters and making choices that impact game progression.

- Story - progresses as part of a structured narrative.

- Task Simulation (Sim) - asks players to perform tasks that are associated with professions, emphasizing the nature of the task.

- Social Sim - asks players to perform tasks associated with social interactions and everyday tasks.

- Collecting - is structured around gathering items for the purpose of having gathered all or as many items as possible.

- Frantic - uses aesthetic elements and/or concurrent game mechanics to saturate the cognitive capabilities of players.

- Chance - progress in the game is largely independent from the actions taken by the player, but differs between game sessions.

\subsection{Procedure}

The survey was conducted over a period of one month during which 117 participants completed the survey. The first part of the survey asked questions about demographics. Participants who never played games were excluded from the study. The second part asked which of 15 predefined games they had played. If two or more games were selected, the next page asked participants to rank the games they had played in terms of how much they had triggered their curiosity while playing. The third part asked participants to provide up to two games (entered as free text) for each of the five curiosity dimensions (see Section 3.1.3). If participants provided two or more items overall, they were asked to rank those games. Participants were free to rank any number of items in both rankings, including none. The final part of the survey was the 5DC questionnaire. Each game provided by participants was assigned two game genres in order of importance. To determine which game genres should be assigned, the two researchers individually assigned game genres in accordance to the definitions (see Section 3.1.5). Assigned game genres were then compared for each game, and disagreements were resolved through discussion. 
Table 1

Game titles mentioned for each of the five curiosity categories (showing titled with at least 5 mentions). Titles in bold appear in multiple categories.

\begin{tabular}{|c|c|}
\hline Game Category & Game Titles (Number of Mentions) \\
\hline GEXP (Explore, find out) 92 unique titles & $\begin{array}{l}\text { Elder Scrolls (17), Fallout (14), Minecraft (11), Zelda: BotW (9), Dark Souls (8), Horizon: Zero Dawn (8), The Witcher (8), } \\
\text { Subnautica (7), World of Warcraft (7), Final Fantasy (5), Assassin's Creed (5), Zelda (5) }\end{array}$ \\
\hline GSOL (Solve) 113 unique titles & Portal (29), The Witness (8), Elder Scrolls (7), Myst (5), The Talos Principle (5) \\
\hline GSAF (Safe, stress-free) 100 unique titles & Sims (8), Stardew Valley (7), Elder Scrolls (6), Cities: Skylines (5) \\
\hline GCON (Connect to people) 84 unique titles & World of Warcraft (13), Final Fantasy (7), Journey (5), Sims (5) \\
\hline GALI (Excited, feeling alive) 108 unique titles & GTA (7), PlayerUnknown's Battlegrounds (6), World of Warcraft (5), Horizon: Zero Dawn (5), Elder Scrolls (5) \\
\hline
\end{tabular}

Data processing A text matching algorithm [37] was used to sort game titles that were entered as free text. Entries belonging to the same game series, or referring to the same game by another name, were combined into a single entry (e.g. Oblivion becomes Elder Scrolls), with the exception of The Legend of Zelda: Breath of the Wild [38] - shortened to Zelda:BotW (separate from The Legend of Zelda game series) and World of Warcraft [39] - shortened to WoW (separate from the Warcraft game series). While this makes it impossible to consider elements of the individual games, games in a series tend to share many of the same general mechanics. This decision allowed us to examine the games over a larger sample size, in service of our exploration of general patterns in game design. Since Zelda: BotW and WoW show significant departures from their predecessors (e.g. going from real-time strategy game to massively multiplayer online role-playing game), these titles were retained.

To identify correlations between the rankings and dimensions on the 5DC questionnaire, three ranks were created for each participant: Predefined selection ranking, game genre ranking, and curiosity category ranking. Predefined selection ranking involved matching the 15 games from the acclaimed games list. Game genre ranking was conducted by assigning every ranked game two genre labels. Since game titles at different ranks could involve the same genres, a TrueSkill rating was calculated for every genre that was part of a participant's ranking. The rating was calculated by comparing all possible genre combinations within a participant's ranking, using both the rank and whether the genre was the primary or secondary label. The genre with the highest rating was ranked 1 , followed by lower rated genres. Genres that were not used received the lowest possible rank of 11 . In addition to creating this ranking for each participant, an overall ranking across all participants was created as well. Curiosity category ranking closely followed the procedure for game genre ranking, with the difference that each game title represented a single category: the category under which the game title was entered. Possible ranks ranged from 1 to 5, reflecting the number of curiosity categories used to represent the five curiosity dimensions of the 5DC questionnaire. Finally, 5DC questionnaire scores were created for each participant by calculating the mean of Likert scale ratings of questions contributing to one of the five dimensions. Likert scale ratings were reverse scored for the Stress Tolerance (ST) dimension, as required by the questionnaire.

\subsection{Key results}

Out of 117 participants, 113 reported playing video games and thus completed the survey (38.9\% female, mean age $\mathrm{M}=27.64, \mathrm{SD}=5.8$ ). The overall ranking of the shared game selection is shown in Fig. 1, with TrueSkill ratings normalized to a $0-1$ range. The count ranged from 19 for Metal Gear Solid V [40] (normalized to 0) to 66 for Portal [41] (normalized to 1). For the game suggestions per category module, a total of 301 unique game titles were mentioned. Table 1 shows which game titles were mentioned most frequently for each of the five game categories. Fig. 3 shows the TrueSkill ranking of the five categories with measures normalized to a $0-1$ range. In terms of counts, GSAF had the fewest game suggestions (103, normalized to 0), and GEXP had the most (180, normalized to 1 ). The overall TrueSkill ranking of game

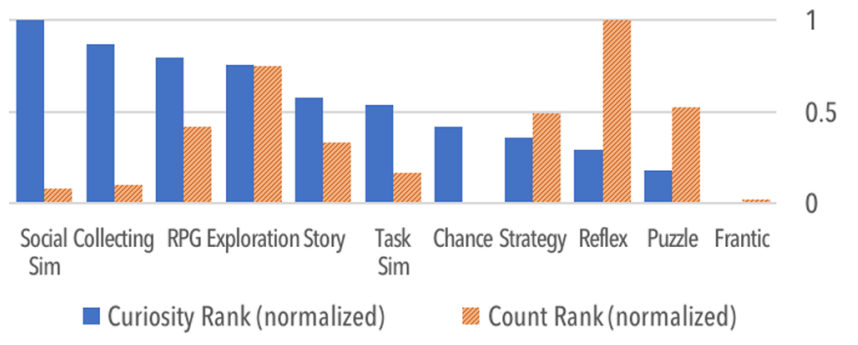

Fig. 2. Game genres ranked by how curious participants felt in games tagged with the genre (blue), and how many games were mentioned for the genre (orange). Values are normalized between 0 and 1. (For interpretation of the references to colour in this figure legend, the reader is referred to the web version of this article.)

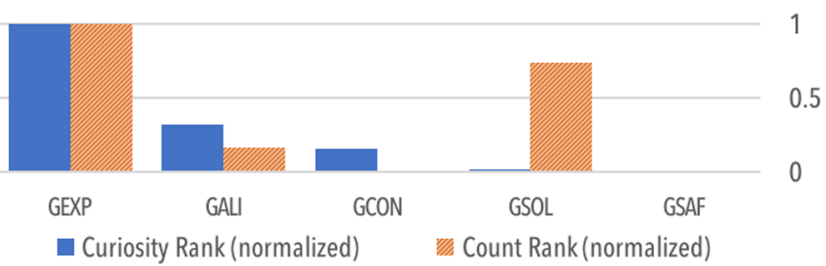

Fig. 3. Curiosity categories based on the 5DC model, ranked by how curious participants felt in games provided under the category (blue), and how many games were mentioned for the category (orange). Values are normalized between 0 and 1 . (For interpretation of the references to colour in this figure legend, the reader is referred to the web version of this article.)

genres associated with games provided by participants is shown in Fig. 2. The frequency of game genres used ranged from 10 for Chance (normalized to 0), to 318 for Reflex (normalized to 1).

The aggregated results of the 5DC questionnaire were: JE $(\mathrm{M}=5.38, \mathrm{SD}=0.86), \mathrm{DS} \quad(\mathrm{M}=4.98, \mathrm{SD}=1.15), \mathrm{ST} \quad(\mathrm{M}=4.36$, $\mathrm{SD}=1.42)$, SC $(\mathrm{M}=5.11, \mathrm{SD}=1.14)$, $\mathrm{TS}(\mathrm{M}=4.20, \mathrm{SD}=1.34)-$ each based on Likert scale ratings from 1 to 7 . We note that we use a significance level of 0.05 for all statistical tests in this study. Significant correlations between 5DC dimensions and rankings are shown in Table 2. For the purpose of clarity, rho was inverted to match the meaning of an increase in score in the individual 5DC dimensions (that is, a rating of 1 in a ranking is 'higher' than a 2, but 1 is lower than 2 in the 5DC questionnaire).

In terms of demographics, playing frequency differed between genders (Mann-Whitney $U=1987, p=0.004$, two-tailed), with male participants playing more frequently. Further differences were found in the ranking of the game genres Strategy ( $U=1911, p=0.002$, twotailed, lower ranking in females) and Task Sim (U $=1714, \mathrm{p}=0.036$, two-tailed, lower ranking in females). Looking at differences in scores of curiosity dimensions, ST was significantly higher in males $(U=978$, $\mathrm{p}=0.001$, two-tailed), while SC was significantly higher in females ( $U=1988, p=0.006$, two-tailed). Participants' age was found to be correlated with a lower score of the 5DC dimension Social Curiosity (rho $=-0.297, \mathrm{p}=0.001$ ), a lower ranking of the game category 
Table 2

Two-tailed Spearman's rank correlations between 5DC dimensions and other measures. VS-MPR shows maximum possible odds in favour of $\mathrm{H}_{1}$ [42].

\begin{tabular}{|c|c|c|c|c|c|}
\hline 5DC Dimension & - & Measure & rho & $\mathrm{p}$ & VS-MPR \\
\hline \multirow[t]{2}{*}{ JE - Joyous Exploration } & - & RPG & 0.2 & 0.037 & 3.019 \\
\hline & - & GCON & 0.234 & 0.016 & 5.617 \\
\hline DS - Deprivation Sensitivity & - & Collecting & -0.193 & 0.045 & 2.655 \\
\hline \multirow[t]{6}{*}{ ST - Stress Tolerance } & - & GTA (IV +) & 0.252 & 0.007 & 10.607 \\
\hline & - & Zelda: BotW & -0.239 & 0.011 & 7.499 \\
\hline & - & Call of Duty & 0.214 & 0.023 & 4.295 \\
\hline & - & RPG & 0.293 & 0.002 & 29.545 \\
\hline & - & Puzzle & -0.226 & 0.018 & 5.037 \\
\hline & - & GSOL & -0.222 & 0.022 & 4.310 \\
\hline \multirow[t]{2}{*}{ SC - Social Curiosity } & - & Social Sim & 0.220 & 0.022 & 4.414 \\
\hline & - & Frantic & -0.212 & 0.03 & 3.535 \\
\hline \multirow[t]{2}{*}{ TS - Thrill Seeking } & - & GTA $($ IV + ) & 0.279 & 0.003 & 22.456 \\
\hline & - & RPG & 0.230 & 0.016 & 5.515 \\
\hline
\end{tabular}

GSAF (rho $=-0.231, \mathrm{p}=0.018$ ), and with a higher ranking of the Puzzle genre (rho $=0.226, \mathrm{p}=0.019$ ).

\subsection{Survey discussion}

Our main goal with this study was to establish game titles and genres that may be of particular interest to the study of curiosity. As an initial point of interest, the defining genres of the higher ranked games in the predefined list are Exploration, Puzzle, RPG, and Reflex. Similarly interesting are games that have not been played by many participants, but ended up high in the ranking nonetheless. Here too, we can see that Exploration and Reflex seem to be involved in games that rank high in curiosity elicitation. For Exploration this is furthermore reflected in the ranking of game categories derived from the 5DC dimensions (see Fig. 3). When asking participants to rank the games they provided, the GEXP category ranked far above other categories, suggesting that exploration and "finding out new things" are considered dominant aspects of what elicits curiosity in a game.

Considering the individual titles on the pre-defined list and correlations with the results of the 5DC questionnaire provided further insight. GTA was ranked higher by participants with increased Stress Tolerance and Thrill Seeking, while Call of Duty [43] (CoD) was ranked higher with increased Stress Tolerance. Given that both GTA and CoD were ranked low overall, this could mean that players do not consider these dimensions as defining of what elicits their curiosity. Zelda:BotW was ranked higher with decreasing ST. Here as well, given the high rating of Zelda:BotW, stress tolerance does not seem to be a predictor of overall curiosity. We speculate that, despite having combat and potentially stressful elements, Zelda:BotW allows players that are easily stressed to still express their curiosity. On the other hand, to express curiosity in GTA or $C o D$, players need a higher stress tolerance.

Participants offered a wide range of games when asked to suggest titles for each of the five curiosity categories. Most of these games gravitated towards one of the five categories (e.g. Minecraft [44] in GEXP). For these games, further analysis towards curiosity invoking design should focus on the theme of the category. Some games (shown in bold) span multiple categories and, for this reason, should be examined in regards to how multiple kinds of curiosity can be motivated in harmony. Turning to the genres of the suggested games, we note several interesting results. Social Sim and Collecting stand out as genres that were part of only a few games, but ended up at the top of the ranking. These genres, and the suggested games that had this genre assigned to them, should be analyzed more closely to see how games can benefit from such elements in terms of increasing their potential to invoke curiosity. Reflex and Puzzle are the opposite of these categories, as they ranked low in curiosity despite being present often. This is noteworthy, as Deprivation Sensitivity specifically deals with puzzle-like stimuli. Games suggested under the corresponding category (GSOL) are mentioned frequently, but ranked low in curiosity (see Fig. 3). It could be that this dimension of curiosity does not strike players as an important component of curiosity. Interestingly, both Zelda:BotW and Portal rank high in the shared game list, despite carrying the Puzzle genre. For these games, it may not be the fact that they include puzzles that invokes curiosity in players. Instead, we hypothesize that exploration is a more defining component in Zelda:BotW, whereas Portal stands out through an unusual base mechanic and surprising narrative components.

Overall, we speculate that game genres that strike a balance between uncertainty and structure tend to rank high, while genres that are highly deterministic (requiring cognitive or physical aptitude) or highly random tend to rank lower in curiosity.

In summary, we found that games involving Exploration, Collecting, and Social Simulation are of particular interest in the further study of curiosity in games. However, as stated previously, the detail of a game's design cannot be captured when it is condensed to two genre labels alone. The next step is therefore to unpack these genres and examine design features present across the games that they were assigned to in order to examine them for their ability to trigger curiosity in players. In the following sections we present a first analysis of the Exploration and Social Simulation genres in order to begin identifying design patterns and related choices that are conductive to eliciting curiosity.

\section{Curiosity in exploration games}

In our survey, games that we attributed to the Exploration genre had the second-highest count of participant-proposed game titles. Considering that Kashdan et al.'s 5DC model understands exploration as one of the five dimensions that make up curiosity, there is a strong case to be made that games that emphasize exploration invoke curiosity in players.

This might seem like an obvious statement. After all, much of the literature on curiosity considers it to be closely linked with exploratory behaviour $[5,45]$. At the same time, the focus tends to be on studying why humans engage in exploration, rather than whether or not the perceived potential for exploration invokes curiosity for doing so. In a fully designed environment, such as in video games, this is a relevant distinction. The mere ability to engage in exploratory behaviour is likely not sufficient to define a game as an Exploration game. Based on the game titles that were mentioned as facilitating exploration, $E x$ ploration games should be defined by their ability to convince players that exploratory behaviour will introduce them to parts of the game that they would not encounter otherwise. This may or may not be the case as far as the actual game design is concerned. On the one hand, it is quite possible to create the illusion of serendipitous encounters while these may actually be prescriptive by the game system. However, if such encounters are rare or are clearly identifiable as disconnected to exploratory behaviour, a game is likely to be attributed to a different genre.

For example, while games in the Portal series seem capable of invoking curiosity, players tend to associate the game more with puzzle solving than exploration. Portal ranked third in the shared selection of games, and was the second-most submitted game title when participants could provide their own suggestions. In Portal, players are tasked with solving tasks that are confined to a relatively small area. For the most part, Portal asks players to figure out the intended solution to a problem. While the game regularly introduces new game elements and surprising narrative turns, both are for the most part identifiable as moments that are intended to happen in a predefined sequence of 
events. Curiosity in Portal is thus more likely connected to the desire to resolve a situation than by the search for novelty.

Even when a game title provides potential for exploration, it may emphasize other game elements, thus making its exploratory aspects less noticeable or less memorable. This could be the case for the GTA game series which ranked 13 out of 15 in the shared game selection and was primarily noted as satisfying the desire for thrill seeking. Despite giving players access to highly detailed environments in which they can explore varied interactions, ranging from performing yoga to playing out simulated drug experiences, the game prioritizes the need for reflexes to make progress. To complete the game, players have to clear several 'missions', most of which are reflex-based or dexterity-based activities such as shooting, driving, or piloting planes. These activities tend to be indicated on a map interface, or are otherwise pointed out by in-game characters, which places less emphasis on exploration. While exploration in GTA is possible and at least partially rewarded in its design, it remains secondary to reflex-based activities.

With these examples in mind, we argue that the mere potential for exploration is not sufficient to characterize an Exploration game in the mind of players. It is the conviction of players that exploratory behaviour will not only be rewarded, but is crucial to progress in the game.

Conceptual and Spatial Exploration. In the design of our survey we defined Exploration games as featuring either spatial or conceptual discovery. Not only is this definition in line with Kashdan et al.'s [13] definition of joyous exploration, it also seemed to suit the existence of games in which exploration can take place through both virtual wandering, as well as epistemic wondering. In the game Spore [46], for example, players are able to shape the evolution of creatures by determining which biological features to 'develop' versus which features to lose. Players are also able to take control of individual creatures and wander through an alien environment. In this way, Spore lets players explore the spatial surroundings, but also gives them the tools to explore a conceptual design space.

While this definition of Exploration games seemed to make sense, the majority of the suggested game titles emphasizes spatial exploration. It is possible that there are simply less game titles that focus on conceptual exploration than on spatial exploration. However, we argue that conceptual exploration is perhaps better captured by the Puzzle game genre and the notion of 'solving', which is what defines the GSOL curiosity category. Within the list of suggested game titles there seemed to be not a single title that was better described by conceptual exploration than by either spatial exploration or the Puzzle genre.

\subsection{Preliminary hypotheses on curiosity in (spatial) exploration games}

Given that the majority of suggested game titles emphasizes specifically spatial exploration, we will focus our analysis on this form of exploration. Based on the list of suggested titles we outline six hypotheses for how curiosity is invoked in spatial Exploration games. These hypotheses are not competing explanations, but rather strategies that may exist parallel to each other.

Where possible we base our hypotheses on related game design patterns that were drafted by Björk and Holopainen [47]. They describe such patterns as a collection of possible design choices in games. Taken together, game design patterns build a 'language' for talking about gameplay; an approach that can be traced to earlier works in architecture and urban design [48], as well as software engineering [49].

Reaching extreme points in the environment. Game environments may feature locations that are difficult to reach. Tall mountains might require a long travel through challenging terrain. Deep trenches may require a dive through dimly lit underwater environments in which players need to manage their oxygen supply. Extreme points can also exist on fairly even ground if other obstacles make it difficult to reach what appears to be a point of interest. Related game design patterns [47] include Inaccessible Areas ("....are parts of the Game World the player can perceive but cannot currently enter, such as areas behind locked doors or sufficiently high ledges"), Outstanding Features (“...are parts of the Game World that cannot be manipulated but by their shape, color, or texture convey information to players."), and to some extent Easter Eggs ("Surprises in the game that are not related to the game"). While explorable areas are by definition accessible, we would argue that it is the uncertainty of whether or not an area is accessible that invokes curiosity in players. Easter Eggs might be defined as unrelated to the game, but players might be enticed to reach extreme points to find out whether Easter Eggs have been hidden at that location. In this case it is not the existence of Easter Eggs that motivates exploration, but the potential that there might be one. What triggers curiosity in reaching extreme points is likely similar to what motivates exploration of extreme points in the real world. Ostensibly, there is little tangible reward in reaching the peak of a mountain or crossing through the scorching heat of a dessert. However, they represent challenges that appear to be just barely possible, and curiosity is invoked by the desire to find out whether this possibility can be realized.

Resolving visual obstructions. Every object within a traversable environment may obstruct visibility of what lies beyond. Games may emphasize such obstructions with the purpose of motivating players to find out what can be found behind such an obstacle. Obstructions may also be artificially imposed, such as through a Fog of War which overlays unexplored territory without the use of in-game objects. Fog of War is in itself a game design pattern mentioned by Björk and Holopainen [47], and is related to the more general pattern of Imperfect Information: "One aspect of information about the total game situation is not fully known to a player, either the information known is totally wrong or the accuracy of the information is limited." While Obstacles are also a common game design pattern, it is not the obstacle itself that invokes curiosity, but rather whatever is obstructed by it. Every object can potentially obstruct something of interest. To invoke curiosity, players need believe that resolving visual obstructions may lead to new and relevant information.

Out of place elements. Games may feature game elements that break an otherwise regular pattern or generally seem out of place in the context of their immediate environment. The game Zelda: BotW features many out of place elements, such as the placement of three identical looking trees where one has an extra apple hanging from a branch. Players can remove the apple to get a small reward, proving to players that interacting with the out of place element triggers a response from the game system (see Fig. 4). What makes this cue work is the fact that trees in the game generally appear to be distributed in a natural way. If the game featured only identical looking trees, it is likely that players would not consider such an occurrence out of place. Cues might be integrated in the environment, such as a trail of tracks, or might come from artificial highlighters such as particle effects. A closely related game design pattern is that of Clues, which "....are game elements that give the players information about how the goals of the game can be reached" as well as the previously mentioned pattern of Imperfect Information [47]. Out of place elements trigger curiosity through the promise of novelty if players interact with them.

Understanding spatial connections. Games that allow players to navigate through an environment may involve complex paths, either through a high degree of interconnectivity or by obfuscating a desired path (e.g. a labyrinth). Another form of asking players to understand spatial connections is by providing hints about a specific location without giving clear instructions how to reach it (e.g. a treasure map or finding out from where a photo has been taken). The closest game design patterns for such sources of curiosity may be found in the aforementioned patterns Outstanding Features, and Imperfect Information [47]. Curiosity is likely invoked by the gap between the mental model a player has about a virtual environment and the actual environment that can be explored by the player.

Foraging for desired objects. Many games feature objects that offer either beneficial effects or are otherwise desirable to obtain. Often such objects are placed in such a way that their discovery is a challenge in 


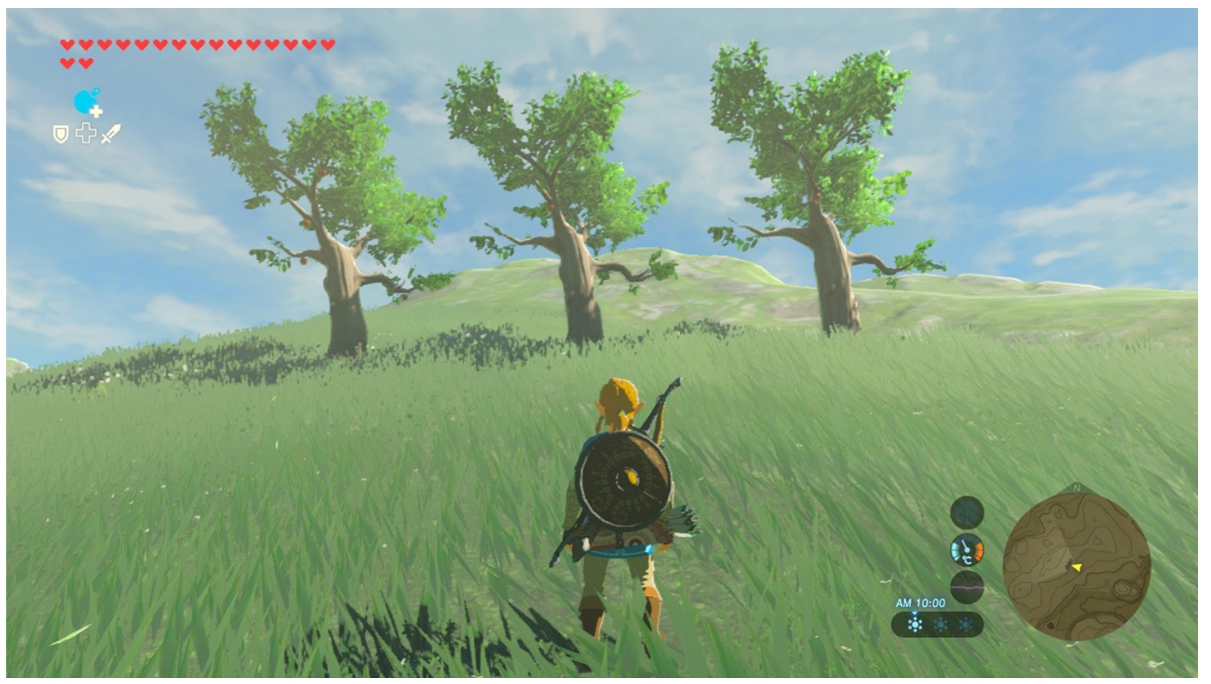

Fig. 4. Screenshot of Zelda: Breath of the Wild (Zelda:BotW), showing three identical-looking trees but with one carrying more apples than the others. Players can pluck the extraneous apples to make the trees fully identical, and thus receive a reward from the game. This is an example of 'out of place elements' that invoke curiosity for spatial exploration. itself. Players are made aware of the existence of such objects and are then asked to look out for them as the game progresses. While the collection of such elements can be considered a motivation in itself (as illustrated by our definition of the Collecting game genre), curiosity might be invoked by asking players to engage in foraging behaviour. When players know that the environment might hold specific objects of interest, they are likely to look for cues that indicate the location of such objects. The word 'object' might suggest a relatively small size, but includes structures that can be entered by the player, as is the case with shrines that are frequently hard to find in Zelda: BotW.

Discovering awe. Exploration in games can be rewarded not only by obtaining a tangible reward, but by the experience of the environment itself. Especially with ever increasing visual fidelity, games seek to provide moments of awe and beauty. Even games with relatively simple graphics can inspire such moments, such as the discovery of a large cave system in Minecraft. Many games create locations that emphasize an interesting vista or a sense of overwhelming scale. In doing so, they attempt to make the experience of the environment rewarding to the player. What invokes curiosity is the promise of finding such places and moments in the game. Once players have been made aware of potentially awe-inspiring moments, they are more likely to be curious for when and where the game might provide a similar experience again.

\section{Curiosity in social simulation games}

Social Simulation games accounted for a relatively small number of game titles that were suggested by participants in our survey. Only games that we attributed to genres Frantic or Chance had fewer titles. Nevertheless, the Social Simulation genre was ranked highest in its ability to invoke curiosity. This means that, on average, games that were classified as Social Simulation games, were ranked higher than games from other genres. Games that were suggested by players under the GCON curiosity category (i.e., games that emphasize understanding of, or connection with other people) also ranked higher than the amount of submitted titles would suggest.

We consider Social Simulation games a specific case of games that involve social actions. These games emphasize the simulation of social actions rather than realized social interactions between people. Simulated social actions may involve directing the everyday tasks of a virtual character, as well as carrying out interactions with other virtual characters. Social Simulation games tend to be played by a single player and thus do not directly facilitate a social connection with other people. While other types of games may establish connections between players and this could be interpreted as social, this does not necessarily mean that players experience a desire to understand those they are playing with. Instead, multiplayer games may quite literally be about connecting with others, but otherwise emphasize game aspects such as reflexes or strategy.

Looking at the game titles that participants suggested under the GCON category, we find some support for considering Social Simulations a subset of games involving social actions. Some of the suggested titles are arguably primarily concerned with connecting players to provide an intelligent or otherwise skilled adversary. Examples for these games include PUBG [50], a shooter game in which 100 players fight each other until a single survivor remains, and Pokémon Go [51], a mobile game that connects players to trade and collect virtual creatures together. Then there are suggestions that lack a direct manner of connecting to others within the game and are intended as single-player experiences, such as The Sims [52], a game in which players manage the lives of several virtual characters, and Life is Strange [53], a choicebased narrative game that tells a supernatural coming-of-age story. Finally, there are game titles for which this distinction is less clear. World of Warcraft is a game in which players ostensibly train a virtual character to defeat both computer-controlled enemies and other players. At the same time, the game gives players many opportunities for social actions that are not immediately in service of defeating an opponent [54]. Another example is Journey [55], a game about wandering through virtual landscapes and coming across other players by serendipity. The game intentionally restricts the mode of communication to simple sounds and character movements, making any encounter with another player a game of signalling intentions. In this sense, Journey encourages reflection on the intentions of the other player due to its design - something which would be lost if a more direct form of communication was made available.

Björk and Holopainen [47] suggest multiple design patterns relating to social interaction within games. However, most patterns do not appear to match well with the dimension of social curiosity defined by Kashdan et al. [13]. For example, patterns related to conflict or trade are mainly concerned with strategic goals, rather than understanding players or entities within the game. Possible exceptions are multiplayer games in which players have competing objectives, in which e.g. patterns of Negotiation or Betrayal are present. In these instances, understanding the thought process of another player is arguably essential to progression in the game. Similarly, while patterns of Collaboration tend to be social in nature, it depends on the design of the game whether understanding of others is motivated through such patterns. The pattern of Team Play suggest the most need for coordination and understanding between players to achieve common goals. However, few of the presented patterns seem to be directly applicable to Social Simulation games. One exception is the pattern of Constructive Play, in which 
players use existing game elements to construct new situations and define their own goals, which partially describes the appeal of games such as The Sims. Overall, patterns related to deeper understanding of game characters or other players, are lacking in the presented collection.

\subsection{Preliminary hypotheses on curiosity in Social Simulation games}

Based on the game titles that were suggested we propose three hypotheses for how Social Simulation games invoke curiosity in players. Given that the genre of Social Simulation in particular ended up high in the ranking, we formulated our hypotheses based on the game titles suggested under GCON that were attributed to that genre, as well as other games of that genre that ended particularly high in the ranking. These hypotheses are not competing explanations but rather strategies that may exist parallel to each other.

Exploration of character depth. Games may involve characters that are either mysterious from the onset, or reveal themselves to be more complex over time. Players may meet other characters in different situations and get to witness different sides of them. This aspect of getting to know a character is frequently woven into the narrative progress of the game, for example in visual novels where players may want to pursue a romantic relationship with another character. In some cases the exploration of character depth extends to the player character as well, as players might find themselves with incomplete information about the motivations of the character they control. What invokes curiosity is the gap between what is already known about a character and the potential depth that could be known.

Engineering social scenarios. Games may give players the ability to arrange the environment of virtual characters to stage social situations. Players may seek to emphasize harmonic cohabitation of characters, or can create dramatic tension and see how the characters react to them. While staged scenarios may appear predictable, the behaviour of game characters are rarely transparent to players. Apart of that, players may invent their own internal narrative for what is happening with characters in the game, without requiring the game system to actually play out such a narrative. Curiosity is thus invoked by giving players the ability to create "What if" scenarios and then seeing how they unfold. It is also possible that players are curious about how they themselves experience observing such scenarios.

Untangling relationships. Social Simulation games may feature a diverse cast of characters that are portrayed as having had pre-existing relationships before the player 'joined'. Relationships between characters may continue to develop throughout the game, or be impacted by actions that were taken by the player. At the beginning of the game, players may have a vague idea about how virtual characters relate to each other and gradually gain a better understanding about the presented relationships. Similar to the promise of individual character depth, curiosity is invoked by bridging the gap between what players know about the link between characters and what can be known.

\section{Study limitations}

Throughout this article, we emphasize the exploratory nature of this study. While we have addressed our reasoning behind specific decisions throughout the article, we find it important to highlight some aspects of our study's design and how they might have impacted our results. In doing so, we aim to help other researchers looking to build upon this work to contextualize the results and shape their own study designs accordingly.

Sampling of participants was done through various online channels, e.g. Facebook groups related to game design and development, as well as mailing lists. The survey was presented in English and so was any mention of it. Due to the choice of the aforementioned recruiting channels and the utilization of our personal and professional network, it is likely that participants were primarily based in western European countries and the USA. We did not gather demographic data of this kind and therefore cannot attest to whether this had an impact on the results. It is reasonable to assume that the same survey conducted in other parts of the world, e.g. Asia, would have generated different results, as other games are likely to be popular in these areas.

Another aspect that is important to reiterate is the inclusion of the TrueSkill algorithm and the decision to have participants provide a ranking of games rather than evaluate them on a Likert scale. While we have outlined our reasoning behind this decision earlier in the article, it is an unconventional measure for evaluating a game's impact on player curiosity. Alternative evaluation methods might provide additional insights while retaining the ability to let participants rank game entries.

Finally, it is likely that detail was lost due to our decision to conflate some of the game titles into games of the same series. Our rationale for this was to examine design patterns across games, rather than focus on specific, individual differences between titles. For future studies, it is important to note that these genre labels require further examination based on the individual game titles that they were assigned to (e.g. through the formulation of design patterns, as presented in this article) in order to understand how they invoke curiosity in players. The takeaway from this study should not be that any game that could be labeled as including exploration automatically invokes curiosity in its players. Design patterns may interact with one another and other elements may be present in a game that work counterproductive to, or are more effective at inducing curiosity. Furthermore, it should be noted that the complexities of individual games are not fully captured by just two genre labels. Further investigations should therefore explore the individual design aspects of the suggested titles and how they invoke curiosity.

\section{Conclusion}

As discussed at the start of this article, there is value in establishing how curiosity can be invoked through design. We take the stance that video games are successful in eliciting curiosity in players and are therefore worth studying for this purpose. To examine how games accomplish this, it is necessary to first understand which existing games manage to invoke curiosity and how they do so as part of their design. Additionally important in formulating this understanding is whether individual tendencies to become curious play a role in which games elicit curiosity in different players. This article presents an initial effort in laying the groundwork to examine these questions.

The first part of this article presents the results of an exploratory survey, in which video game titles and genres were gathered and ranked in regards to how much they were able to invoke curiosity in players. From examining the survey results, we speculate that games that strike a balance between uncertainty and structure tend to rank high in their ability to invoke curiosity, while highly deterministic or highly random games tend to rank lower. The first contribution of this article is the ranking of particular game titles and genres. We further establish how these rankings correspond to different types of trait curiosity in players and provide suggestions to which game design aspects require more comprehensive study. These results do not provide a definitive answer as to which games make players curious and how. However, they provide a tangible direction for furthering the knowledge of how games invoke curiosity and highlight considerations to note for future studies.

The second part of the article ventures into suggesting design patterns that invoke curiosity within two highly ranked game genres from the survey - Exploration and Social Simulation. To produce generally applicable theory as to how games may be designed to elicit curiosity in players, it is necessary to look beyond individual features of particular games and examine overarching patterns of game design that may be implemented within various contexts. The game design patterns presented in this paper were thus formulated by analyzing the design of multiple game titles that were assigned the Exploration and Social Sim 
genres. In describing these patterns, this article furthers the analysis of these specific game genres. We emphasize that these are hypothetical in nature and require empirical validation through follow-up experiments to examine whether they are indeed effective in inducing curiosity and why. We expect these hypotheses to be extended upon and amended through future studies, for which this article provides a starting point. Once more specific design patterns have been identified as being capable of invoking curiosity, they should then be examined in further detail. For example, studies may investigate whether they remain effective when introduced into games that emphasize lower ranked game genres, or whether their efficacy depends on the involvement of specific genres.

Ultimately, research efforts in this area should aim for the formulation of guidelines to design for curiosity. By fostering the development of games that intentionally invoke curiosity, it may be possible to increase an individual's disposition to become curious. Such games would also provide interactive environments through which curiosity and related behaviour can be studied in the laboratory. Whether this potential can be fulfilled remains to be seen, but this work provides a concrete basis for continued exploration of this topic.

\section{Declaration of Competing Interest}

We hereby state that there is no conflict of interest with the submission of this manuscript. The authors have no affiliation with any organization with a direct or indirect financial interest in the subject matter discussed in the manuscript.

\section{Acknowledgements}

We would like to thank all participants of this study for their time and care, as well as Max van Duijn for his mentorship and support.

\section{Appendix A. Supplementary material}

Supplementary data associated with this article can be found, in the online version, at https://doi.org/10.1016/j.entcom.2019.100320.

\section{References}

[1] J.A. Litman, Interest and deprivation factors of epistemic curiosity, Personal. Individ. Differ. 44 (7) (2008) 1585-1595.

[2] J.A. Litman, Relationships between measures of i-and d-type curiosity, ambiguity tolerance, and need for closure: an initial test of the wanting-liking model of information-seeking, Personality Individ. Differ. 48 (4) (2010) 397-402.

[3] T.G. Reio Jr, J.M. Petrosko, A.K. Wiswell, J. Thongsukmag, The measurement and conceptualization of curiosity, J. Genet. Psychol. 167 (2) (2006) 117-135.

[4] F.F. Schmitt, R. Lahroodi, The epistemic value of curiosity, Educ. Theory 58 (2) (2008) 125-148.

[5] E.M. Grossnickle, Disentangling curiosity: dimensionality, definitions, and distinctions from interest in educational contexts, Educ. Psychol. Rev. 28 (1) (2016) 23-60.

[6] G. Loewenstein, The psychology of curiosity: a review and reinterpretation, Psychol. Bull. 116 (1) (1994) 75.

[7] G. Pluck, H. Johnson, Stimulating curiosity to enhance learning, GESJ, Educ. Sci. Psychol. 2 (19) (2011) 1512-1801.

[8] M.J. Kang, M. Hsu, I.M. Krajbich, G. Loewenstein, S.M. McClure, J.T.-Y. Wang, C.F. Camerer, The wick in the candle of learning: epistemic curiosity activates re ward circuitry and enhances memory, Psychol. Sci. 20 (8) (2009) 963-973.

[9] A. To, A. Safinah, G.F. Kaufman, J. Hammer, Integrating curiosity and uncertainty in game design, Proceedings of 1st International Joint Conference of DiGRA and FDG, 2016, pp. 1-16.

[10] M. Schaekermann, G. Ribeiro, G. Wallner, S. Kriglstein, D. Johnson, A. Drachen, R. Sifa, L.E. Nacke, Curiously motivated: profiling curiosity with self-reports and behaviour metrics in the game destiny, Proceedings of the Annual Symposium on Computer-Human Interaction in Play, ACM, 2017, pp. 143-156.

[11] A. To, J. Holmes, E. Fath, E. Zhang, G. Kaufman, J. Hammer, Modeling and designing for key elements of curiosity: risking failure, valuing questions, Trans. Digital Games Res. Assoc. 4 (2) (2018) 138-171.
[12] J. Jirout, D. Klahr, Children's scientific curiosity: in search of an operational definition of an elusive concept, Dev. Rev. 32 (2) (2012) 125-160.

[13] T.B. Kashdan, M.C. Stiksma, D.D. Disabato, P.E. McKnight, J. Bekier, J. Kaji, R. Lazarus, The five-dimensional curiosity scale: capturing the bandwidth of curiosity and identifying four unique subgroups of curious people, J. Res. Pers. 73 (2018) 130-149.

[14] I. Inan, The Philosophy of Curiosity, Routledge, 2013.

[15] J. Dewey, How We Think, Heath \& Co., 1910

[16] D.E. Berlyne, A theory of human curiosity, Br. J. Psychol. 45 (3) (1954) 180-191.

[17] D.E. Berlyne, Conflict, Arousal, and Curiosity, McGraw-Hill Book Company, 1960.

[18] C.D. Spielberger, L.M. Starr, Curiosity and exploratory behavior, Motiv. Theor. Res. (1994) 221-243.

[19] J.A. Litman, P.J. Silvia, The latent structure of trait curiosity: evidence for interest and deprivation curiosity dimensions, J. Pers. Assess. 86 (3) (2006) 318-328.

[20] J.A. Litman, R.P. Collins, C.D. Spielberger, The nature and measurement of sensory curiosity, Personality Individ. Differ. 39 (6) (2005) 1123-1133.

[21] T.B. Kashdan, J.E. Roberts, Trait and state curiosity in the genesis of intimacy: differentiation from related constructs, J. Soc. Clin. Psychol. 23 (6) (2004) 792.

[22] T.G. Reio, J.L. Callahan, Affect, curiosity, and socialization-related learning: a path analysis of antecedents to job performance, J. Bus. Psychol. 19 (1) (2004) 3-22.

[23] J.A. Litman, T.L. Jimerson, The measurement of curiosity as a feeling of deprivation, J. Pers. Assess. 82 (2) (2004) 147-157.

[24] H.I. Day et al., Intrinsic motivation: A new direction in education.

[25] E. McAuley, T. Duncan, V.V. Tammen, Psychometric properties of the intrinsic motivation inventory in a competitive sport setting: a confirmatory factor analysis, Res. Quart. Exercise Sport 60 (1) (1989) 48-58.

[26] M. Zuckerman, The sensation seeking scale v (sss-v): still reliable and valid, Personal. Individ. Differ. 43 (5) (2007) 1303-1305.

[27] G. Costikyan, Uncertainty in Games, Mit Press, 2013.

[28] C. Klimmt, Dimensions and determinants of the enjoyment of playing digital games: a three-level model, in: Level up: Digital Games Research Conference, 2003, pp. 246-257.

[29] L.E. Nacke, C. Bateman, R.L. Mandryk, Brainhex: preliminary results from a neurobiological gamer typology survey, International Conference on Entertainment Computing, Springer, 2011, pp. 288-293.

[30] S. Kreitler, E. Zigler, H. Kreitler, The nature of curiosity in children, J. Sch. Psychol. 13 (3) (1975) 185-200.

[31] Metacritic.com, Page: Best video games of all time, 2018. http://www.metacritic. com/browse/games/score/metascore/all/all/filtered (accessed: 2018-01-31).

[32] G.N. Yannakakis, H.P. Martínez, Ratings are overrated!, Front. ICT 2 (2015) 13.

[33] H. Lee, Trueskill 0.4.4 python package, 2015. http://trueskill.org/.

[34] R. Herbrich, T. Minka, T. Graepel, Trueskill(tm): A Bayesian Skill Rating System, MIT Press, 2007 pp. 569-576. URL https://www.microsoft.com/en-us/research/ publication/trueskilltm-a-bayesian-skill-rating-system/.

[35] R.I. Clarke, J.H. Lee, N. Clark, Why video game genres fail: a classificatory analysis, Games Culture 12 (5) (2017) 445-465.

[36] Rockstar North Ltd., Grand Theft Auto V, [PlayStation 3/4, Windows, Xbox 360/ One], 2013.

[37] SeatGeek, Fuzzywuzzy, 2017. https://github.com/seatgeek/fuzzywuzzy.

[38] Nintendo EPD, The Legend of Zelda: Breath of the Wild, [Nintendo Switch, Wii U], 2017.

[39] Blizzard Entertainment Inc., World of WarCraft, [Macintosh, Windows], 2004.

[40] Kojima Productions, Metal Gear Solid V: The Phantom Pain, [PlayStation 3/4, Windows, Xbox 360/One], 2015.

[41] Valve Corporation, Portal, [Android, Linux, Macintosh, Windows], 2007.

[42] T. Sellke, M. Bayarri, J.O. Berger, Calibration of $\rho$ values for testing precise null hypotheses, Am. Stat. 55 (1) (2001) 62-71.

[43] Infinity Ward Inc, Call of Duty 4: Modern Warfare, [Macintosh, PlayStation 3, Wii, Windows, Xbox 360], 2007.

[44] A.B. Mojang, Minecraft, [Browser, Linux, Macintosh, Windows], 2010.

[45] J. Stagl, A History of Curiosity: The Theory of Travel 1550-1800, Routledge, 2012.

[46] Maxis Software Inc., Spore, [Macintosh, Windows] (2008).

[47] S. Bjork, J. Holopainen, Patterns in game design (game development series).

[48] C. Alexander, A Pattern Language: Towns, Buildings, Construction, Oxford University Press, 1977.

[49] E. Gamma, R. Helm, R. Johnson, J. Vlissides, Design patterns: elements of reusable object-oriented software.

[50] Bluehole Ginno Games, PlayerUnknown's Battlegrounds, [Android, iOS, PlayStation 4, Windows, Xbox One], 2017.

[51] Niantic Inc., Nintendo Co., Ltd., The Pokémon Company, Pokémon GO, [Android, iOS], 2016.

[52] The Sims Studio, The Sims 4, [Macintosh, PlayStation 4, Windows, Xbox One], 2014.

[53] DONTNOD Entertainment, Life Is Strange, [Android, iOS, Linux, Macintosh, PlayStation 3/4, Windows, Xbox 360/One], 2015

[54] V.H. Chen, H.B. Duh, Understanding social interaction in world of warcraft, Proceedings of the International Conference on Advances in Computer Entertainment Technology, ACM, 2007, pp. 21-24.

[55] thatgamecompany, LLC, Journey, [PlayStation 3/4], 2012. 